\title{
Development and Implementation of Smart Home Monitoring System in Embedded Environment
}

\author{
Jie Zhang \\ School of Electrical Engineering, Chengdu Textile College, Chengdu, China \\ 3047699076@qq.com
}

Keywords: Embedded; Smart home; monitoring system

\begin{abstract}
With the rapid development of computer technology, sensor technology, communication technology, smart home has become the development trend of the future life, it will be all the items in the family connected by a network, the realization of the home environment remote monitoring and intelligent management, so as to create a safe, comfortable and convenient living environment for the people. Taking intelligent home control system function, safety as the starting point, the design of smart home remote monitoring system based on embedded environment. The system can meet the real-time monitoring to the home situation of the people, and remote control of household equipment through the network, has the security alarm, remote control, remote environmental monitoring and other functions, and has certain practical significance.
\end{abstract}

\section{Introduction}

With the rapid development of information technology, people to be resided to the home life of comfort, convenience, higher and higher demands are proposed, and hope that in the process of going out, you can remote view the various states in the home, and can undertake the corresponding control of household equipment, so that they can safely travel, and enjoy the comfortable home life, fully realize the benefits. This article is basic premise with safe, practical, build a smart home based on embedded Linux development platform overall design scheme of remote monitoring system. The system has a family security alarm, household appliances remote control, remote monitoring environment three big functions, realizes the user for the intelligent management of household. Practical tests show that the system is running well, can realize the expected function, to meet the design requirements.

\section{Smart home}

Smart home, also known as the intelligent residential, it is the use of computer, network communication and other advanced technology, will be a variety of related subsystems Home Furnishing life together in a certain way, unified management, the Home Furnishing life more comfortable, convenient, safe. Home Furnishing intelligent control system is a set of visual intercom, home security alarm, environmental monitoring, remote control, remote video monitoring, remote network education, community service, home entertainment and many seed system in one multi-functional integrated system. Its main purpose is the existence of all kinds of family and information communication equipment, home appliances and related Home Furnishing security device through the network, the right way, connected to an intelligent system, so as to carry out centralized or remote monitoring, control and management of affairs Home Furnishing.

\section{The design of the overall scheme of intelligent Home Furnishing monitoring system}

The overall framework. Smart home is a monitoring, control, analysis, service and management function of the complex, is the organic integration of computer technology, wireless communication technology and electronic sensor technology, the purpose is to let the user to get the 
full range of services, the timely for real-time processing of home environment parameter monitoring and information, as shown in Figure 1.

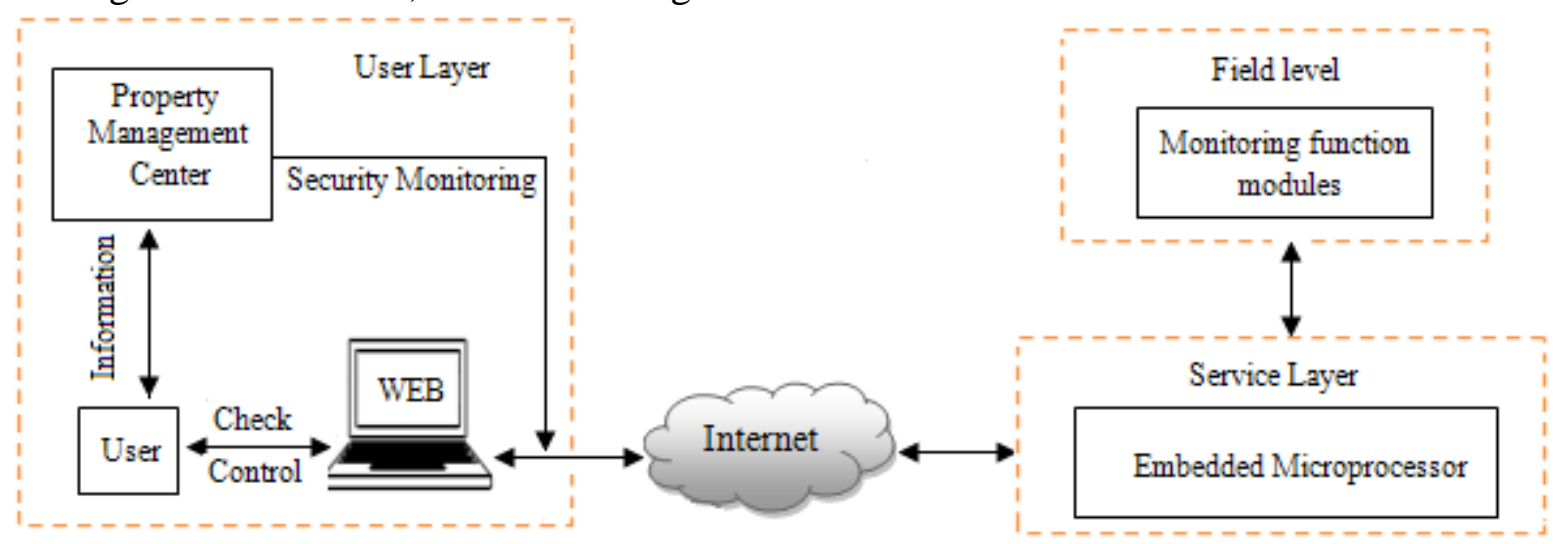

Fig.1 Architectural structure

Main function. In this paper, the design of the embedded intelligent household monitoring system is mainly used to realize the family security alarm, household appliances remote control, remote monitoring environment three big functions, specific function is shown in figure 2.

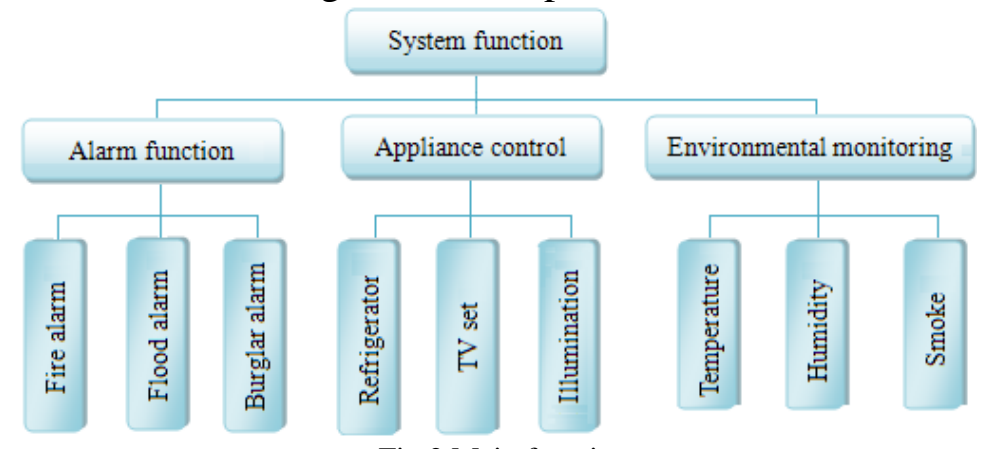

Fig.2 Main function

Security alarm module: Sensor technology was used to fire, leakage of the family, the circumstance such as personnel into the real-time monitoring, once found, the scene of the alarm rang, immediately and will alert through cell phone messages sent to the residents.

In electrical home appliances control module: The family refrigerator, TV, air conditioning, lighting and other home appliances in the open or closed monitoring, and can through the remote control which changes its current state.

Environmental monitoring module: By sensors for temperature, humidity, each room in the family and the kitchen smoke to monitor the situation.

\section{Design and implementation of intelligent household monitoring system}

\section{Hardware design.}

1) Home gateway based on ARM processor platform.

In the embedded environment, common control chip ARM, such as MCU, DSP, FPGA, corresponding to their respective applications. In this system, the need to drive to the various bus and I/o devices, as well as the need to build an embedded Linux operating system, and need to run on an embedded web server and embedded database, so the system USES a function is more powerful, the power consumption of a relatively small ARM processor as the core control unit. This system at the core of the processor USES a SAMSUNG S3C2440 chip, the chip has a low price, high performance, low consumption, and many other characteristics, make its can better support Linux embedded operating system.

2) Infrared transceiver module circuit design

The core control chip is enhanced based on AVR RISC structure of low-power 8-bit CMOS microcontroller. Is mainly composed of infrared light-emitting diode and matching circuit, can emit infrared wavelength of $940 \mathrm{~nm}$ or so. With $38 \mathrm{KHZ}$ carrier signal modulation, the infrared coding 
sequence modulation of the output signal after amplification, drive the infrared light-emitting diode, needed to get the infrared emission signal, principle as shown in figure 3.

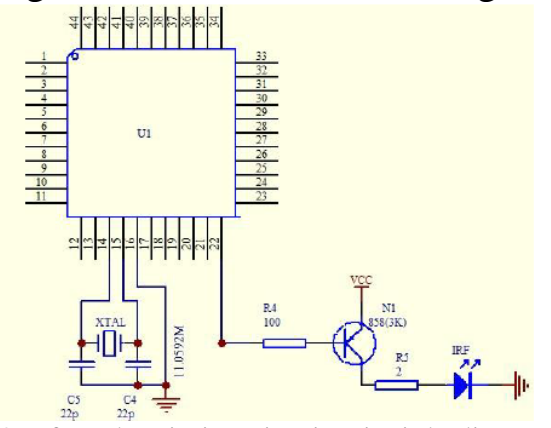

Fig.3 Infrared emission circuit principle diagram

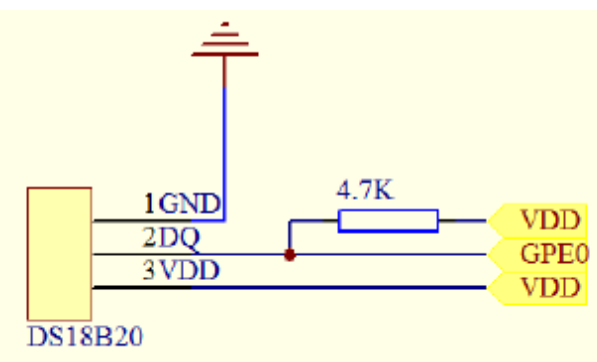

Fig.4 Connecting way of DS18B20 and ARM

3) Temperature acquisition circuit design

The system temperature acquisition module of temperature sensor is relatively common in the market produced by DALLAS company DS18B20, with strong anti-interference ability, high precision, packaging small volume, simple installation and so on, can be easily connected to the ARM processor, connection as shown in figure 4 .

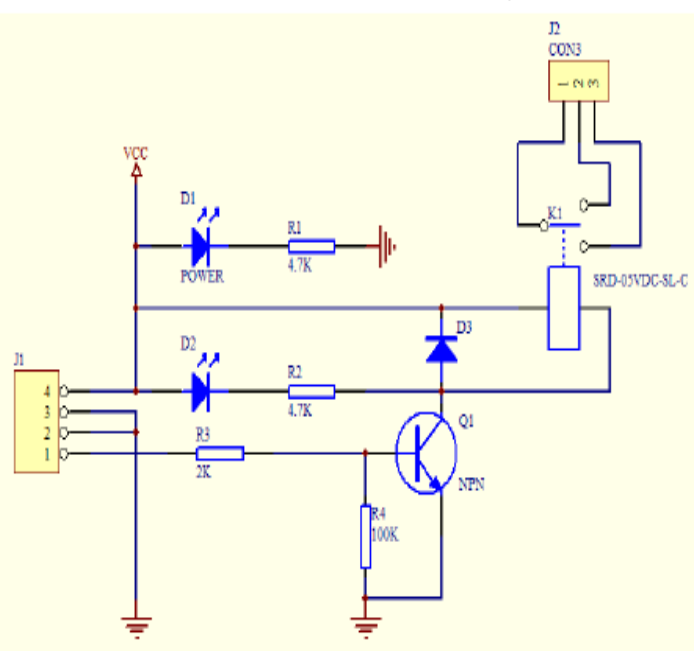

Fig.5 Relay drive circuit

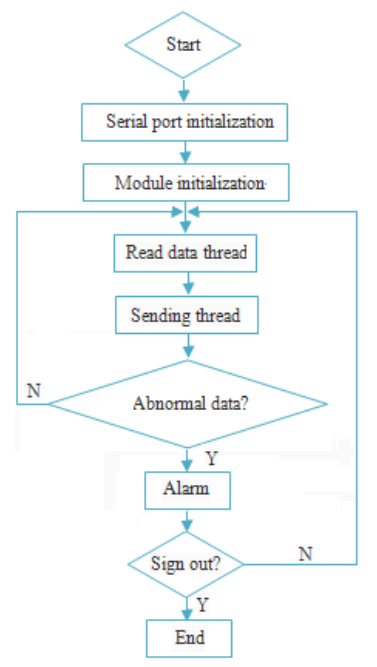

Fig.6 Program flow diagram

4) Lighting electrical relay drive circuit

This system adopts the processor GPIO pin as a relay drive circuit input, through the input level of high and low to control the relay coil and absorption or not, and then complete the switch control of household lighting equipment, circuit diagram as shown in figure 5.

\section{The software design.}

1) Embedded Web server selection

Based on embedded devices running when the effective resources and space, should choose to take up fewer resources and can be a very good response to user requests a Web server. Boa is a powerful server that has the characteristics of support CGI authentication, source code is open source, runtime only very little memory to meet the requirements, very suitable for application in the embedded devices. The embedded Web server system adopts the Boa server.

2) Server application design

Web server is not only to implement the data through the Web page background program and user layer interaction, also need to implement the serial communication between the control panel and alarm. Under Linux, this design is based on the $\mathrm{C}$ language to complete the server application development, program flow is shown in figure 6.

Test. Through the practical application to verify the system, only to the room temperature monitor. In different room temperature sensor for monitoring the scene temperature acquisition, monitoring the test results through the server, as shown in figure 7. 


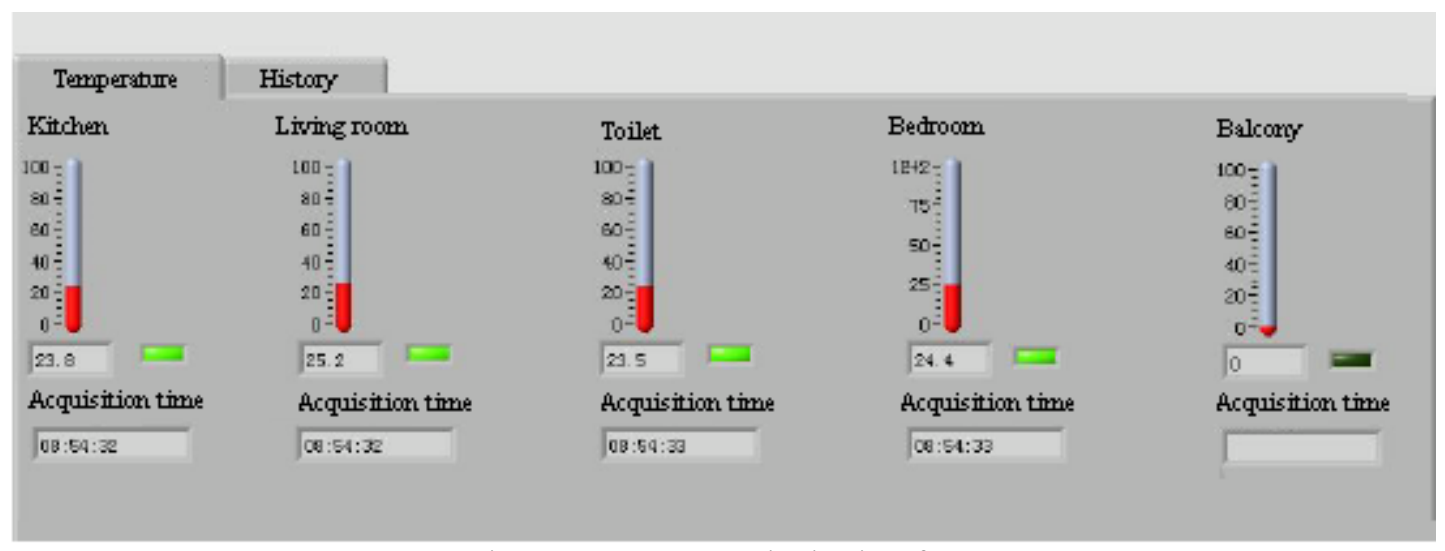

Fig.7 Temperature monitoring interface

Monitoring software monitoring data uploaded into temperature value display. If a node receives the monitoring data upload, the exhibit of lanterns lit status indicator, indicating that the activation status of the node in the. Contains the data acquisition time to upload data parsed in the monitoring interface, real-time display.

\section{Conclusion}

The future smart home is an inevitable development trend of human home life, it will be advanced technology into people's daily home life, greatly improve the people's living comfort and sense of security. The realization principle and applications of embedded intelligent home based on detailed research, overall design scheme of intelligent home remote monitoring system based on embedded Linux development platform; through the reasonable selection of embedded intelligent home monitoring system software and hardware and design, realized the family security alarm, remote control, remote environmental monitoring function, realize user intelligent management of home equipment, the experiments show that the system can achieve the design function, which provides a reference for the design of intelligent home control system.

\section{References}

[1] Bar-Shalom, F. Daum, J. Huang. The Probabilistic Data Association Filter [J]. IEEE Control Systems Magazine. 2009, 29(6): 82 100

[2] Y. W. Mo, D.Y. Xiao. Hybrid System Monitoring and Diagnosing Nosed on Particle Filter Algorithm. Acta Automation Sonica. 2003, 29(3): 641 648

[3] Vicaire P, He T, Cao Q, et al. Achieving long-term surveillance in Vigil Net[J] .ACM Transactions on Sensor Networks ( TOSN) , 2009, 5(1):1-39

[4] Z hai Y, Y eary B. A new centralized sensor fusion tracking methodology based on particle filtering for power aware systems. IEEE Transactions on Instrumentation and Measurement, 2008,57(10): 2377- 2387

[5] P. Bao. Localization Algorithm Based on Sector Scan for Mobile Wireless Sensor Networks [J]. Applied Mathematics \& Information Sciences. 2012, 6: 99-103 